\title{
Association between ZFHX3 and PRRX1 Polymorphisms and Atrial Fibrillation Susceptibility from Meta-Analysis
}

\author{
Liting Wu $\mathbb{D}$, Min Chu $\mathbb{D}$, and Wenfang Zhuang \\ Medical Laboratory, Shidong Hospital, Affiliated to University of Shanghai for Science and Technology, No. 999, \\ Shiguang Road 200438, Yangpu District, Shanghai, China \\ Correspondence should be addressed to Wenfang Zhuang; czwf1991@163.com
}

Received 15 August 2021; Accepted 15 October 2021; Published 14 December 2021

Academic Editor: Lanfranco D Elia

Copyright (c) 2021 Liting Wu et al. This is an open access article distributed under the Creative Commons Attribution License, which permits unrestricted use, distribution, and reproduction in any medium, provided the original work is properly cited.

Background. Atrial fibrillation (AF) is a common, sustained cardiac arrhythmia. Recent studies have reported an association between ZFHX3/PRRX1 polymorphisms and AF. In this study, a meta-analysis was conducted to confirm these associations. Objective and Methods. The PubMed, Embase, and Wanfang databases were searched, covering all publications before July 20 , 2020. Results. Overall, seven articles including 3,674 cases and 8,990 healthy controls for ZFHX3 rs2106261 and 1045 cases and 1407 controls for PRRX1 rs3903239 were included. The odds ratio (OR) (95\% confidence interval (CI)) was used to assess the associations. Publication bias was calculated using Egger's and Begg's tests. We found that the ZFHX3 rs2106261 polymorphism increased AF risk in Asians (for example, allelic contrast: OR [95\% CI]: 1.39 [1.31-1.47], $P<0.001$ ). Similarly, strong associations were detected through stratified analysis using source of control and genotype methods (for example, allelic contrast: OR [95\% CI]: 1.51 [1.38-1.64], $P<0.001$ for HB; OR [95\% CI]: 1.31 [1.21-1.41], $P<0.001$ for PB; OR [95\% CI]: 1.55 [1.33-1.80], $P<0.001$ for TaqMan; and OR [95\% CI]: 1.31 [1.21-1.41], $P<0.001$ for high-resolution melt). In contrast, an inverse relationship was observed between the PRRX1 rs3903239 polymorphism and AF risk (C-allele vs. T-allele: OR [95\% CI]: 0.83 [0.77-0.99], $P=0.036$; CT vs. TT: OR [95\% CI]: 0.79 [0.67-0.94], $P=0.006)$. No obvious evidence of publication bias was observed. Conclusions. In summary, our study suggests that the ZFHX3 rs2106261 and PRRX1 rs3903239 polymorphisms are associated with AF risk, and larger casecontrols must be carried out to confirm the abovementioned conclusions.

\section{Introduction}

Atrial fibrillation (AF) is a common form of arrhythmia, with an incidence of approximately $1 \%$ among adults worldwide $[1,2]$. Previous studies have demonstrated that AF significantly increases the social and economic burden in both developed and developing countries [3]. Additionally, $\mathrm{AF}$ is the main cause of heart failure and stroke $[4,5]$. A variety of structural heart diseases and systemic diseases are related to $\mathrm{AF}$, including congestive heart failure, cardiomyopathy, pulmonary heart disease, essential hypertension, and hyperthyroidism $[6,7]$, while age, obesity, smoking, excessive drinking, and drug use also contribute to the development of AF $[6,8]$. Thus far, the exact pathogenesis of AF remains unclear. However, many studies have suggested that genetic factors play an important role in AF occurrence and development [9]. In fact, common genetic variants (a multitude of single-nucleotide polymorphisms (SNPs)) associated with AF have been detected in genome-wide association studies (GWASs) [10-12], such as endothelial nitric oxide synthase 786 T/C, CYP11B2 rs1799998, KCNE1 G38S, and caveolin-1 rs3807989 [9, 13-15].

Two independent GWASs identified significant associations between rs2106261 and rs7193343 polymorphisms in the zinc finger homeobox 3 (ZFHX3) gene and AF susceptibility in various populations of European ancestry $[16,17]$. ZFHX3 is located on chromosome 16q22. Benjamin et al. [16] indicated that the rs2106261 SNP in ZFHX3 was associated with $\operatorname{AF}\left(\mathrm{OR}=1.19 ; P=2.76 \times 10^{-7}\right)$. At the same time, Gudbjartsson et al. [17] assessed another SNP (rs7193343) in ZFHX3, which was confirmed to be related to $\mathrm{AF}$ in Icelandic individuals $\left(\mathrm{OR}=1.21, P=1.4 \times 10^{-10}\right)$. 
Paired homeobox 1 (PRRX1) encodes a homeodomain transcription factor that is highly expressed in the developing heart [18]. Fetal lung vascular development was impaired in a PRRX1 knockout mouse model [19]. The expression pattern of PRRX1 in the mouse atria was evaluated; both genes were overexpressed in the left atrium when compared to the right atrium [20]. These results suggest that PRRX1 may play a vital role in heart diseases, including AF. In a subsequent meta-GWAS, the PRRX1 rs3903239 variant was associated with $\mathrm{AF}$ risk $\left(P=8.4 \times 10^{-14}\right)$ [21].

Taking into consideration the more precise assessment of the ZFHX3 rs2106261 and PRRX1 rs3903239 variants in AF risk, we must first perform a meta-analysis of all eligible case-control studies to confirm the associations [18, 22-27].

\section{Materials and Methods}

2.1. Identification and Eligibility of Relevant Studies. The PubMed, Embase, and Wanfang databases were selected. The last search was conducted on July 20, 2020, with the search terms including the keywords "ZFHX3" or "zinc finger homeobox 3," "PRRX1" or "paired related homeobox 1," "polymorphism" or "variant," and "atrial fibrillation." After the abovementioned search, a total of 96 publications were identified, of which 7 met the inclusion criteria.

2.2. Criteria for Inclusion and Exclusion. The studies included in the analysis met all of the following conditions: (a) the study assessed the correlation between AF and the ZFHX3 rs2106261 polymorphism and/or PRRX1 rs3903239 polymorphism; (b) unpaired case-control studies; and (c) sufficient genotypes in cases and controls. In addition, the following exclusion criteria were applied: (a) no control group; (b) no genotype frequency was available; and (c) previous publications were repeated.

2.3. Data Extraction. Two of the authors extracted all data independently and complied with the selection criteria. The following items were collected: author's name, ethnicity, year of publication, total of each genotype case/control number, country, source of control, genotyping methods, and Hardy-Weinberg equilibrium (HWE) of controls.

2.4. Quality Score Assessment. The Newcastle-Ottawa Scale (NOS) was used to assess the quality of each study and evaluate all aspects of the methodology, including case selection, comparability between groups, and exposure determination. The NOS has a total score of 0-9 stars. Research with a score greater than 7 is considered a high-quality study [28].

2.5. Statistical Analysis. Based on the genotype frequencies of the cases and controls, the probability odds ratio (OR) with $95 \%$ confidence interval (CI) was used to measure the strength of the association between the polymorphisms and AF. First, we conducted a subgroup analysis stratified by race. The source of the control subgroup analysis was carried out in two categories: population based (PB) and hospital based (HB).

The statistical significance of the OR was determined using the $Z$-test. The fixed and random effect models were used to calculate the combined OR. The $Q$-test $(P \geq 0.10)$ indicated heterogeneity between the included studies. If significant heterogeneity was detected, the random-effects model (DerSimonian-Laird method) was used, but otherwise, the fixed-effects model (Mantel-Haenszel method) was selected [29, 30]. For ZFHX3 rs2106261, we investigated the relationship between genetic variants and AF risk in allelic contrast (A-allele vs. G-allele), homozygote comparison (AA vs. GG), the dominant genetic model $(\mathrm{AA}+\mathrm{AG} v s . \mathrm{GG})$, heterozygote comparison (AG vs. GG), and recessive genetic models (AA vs. $A G+G G$ ). For PRRX1 rs3903239, C-allele vs. T-allele, CT vs. TT, CC vs. $\mathrm{TT}, \mathrm{CC}+\mathrm{CT} v s$. TT, and CC vs. CT + TT models were applied. Funnel plot asymmetry was assessed using Begg's test, and publication bias was assessed using Egger's test [31]. The departure of frequencies from expectation under HWE was assessed using the $\chi^{2}$ test in the controls through the Pearson chi-square test $(P<0.05$ was considered significant) [32]. All statistical tests for this meta-analysis were performed using Stata software (version 11.0; StataCorp LP, College Station, TX, USA).

2.6. ZFHX3 and PRRX1 Interaction Networks. To fully understand the role and potential functional partners of ZFHX3 and PRRX1 in AF, the String online server (https:// string-db.org/) was used to create a gene-gene interaction network of ZFHX3 and PRRX1 [33].

\section{Results}

3.1. Eligible Studies. In total, 96 articles were collected from the PubMed, Embase, and Wanfang databases. Of these, 89 articles were excluded (25 unrelated articles, 4 systematic/metaanalysis studies, 1 with only a case group, 23 supplements, 30 duplications, and 6 with no original numbers for case/control groups) (Figure 1). Finally, seven articles were identified in the current analysis, including 3,674 cases and 8,990 healthy controls related to the ZFHX3 rs2106261 polymorphism and 1045 cases and 1407 controls for the PRRX1 rs3903239 polymorphism. The characteristics of each study are presented in Table 1. In addition, the minor allele frequency (MAF) reported from the five main worldwide populations in the 1000 Genomes Browser were checked (https://www.ncbi.nlm.nih. gov/snp/): African, European, East Asian, American, and South Asian populations (Figure 2); the MAF was similar to the average level in our current case and control groups.

3.2. ZFHX3 rs2106261 and AF Risk. In the overall analysis, increased associations were observed in five genetic models in Asians: allelic contrast (OR [95\% CI] = 1.39 [1.31-1.47], $P_{\text {heterogeneity }}=0.117, P<0.001$, Figure $\left.3(\mathrm{a})\right)$, heterozygote comparison $\quad(\mathrm{OR} \quad[95 \% \quad \mathrm{CI}]=1.37 \quad[1.18-1.59]$, $P_{\text {heterogeneity }}=0.007, P<0.001$, Figure $\left.3(\mathrm{~b})\right)$, AA $v s$. CC (OR $[95 \% \mathrm{CI}]=1.96[1.73-2.21], P_{\text {heterogeneity }}=0.317, P<0.001$, 


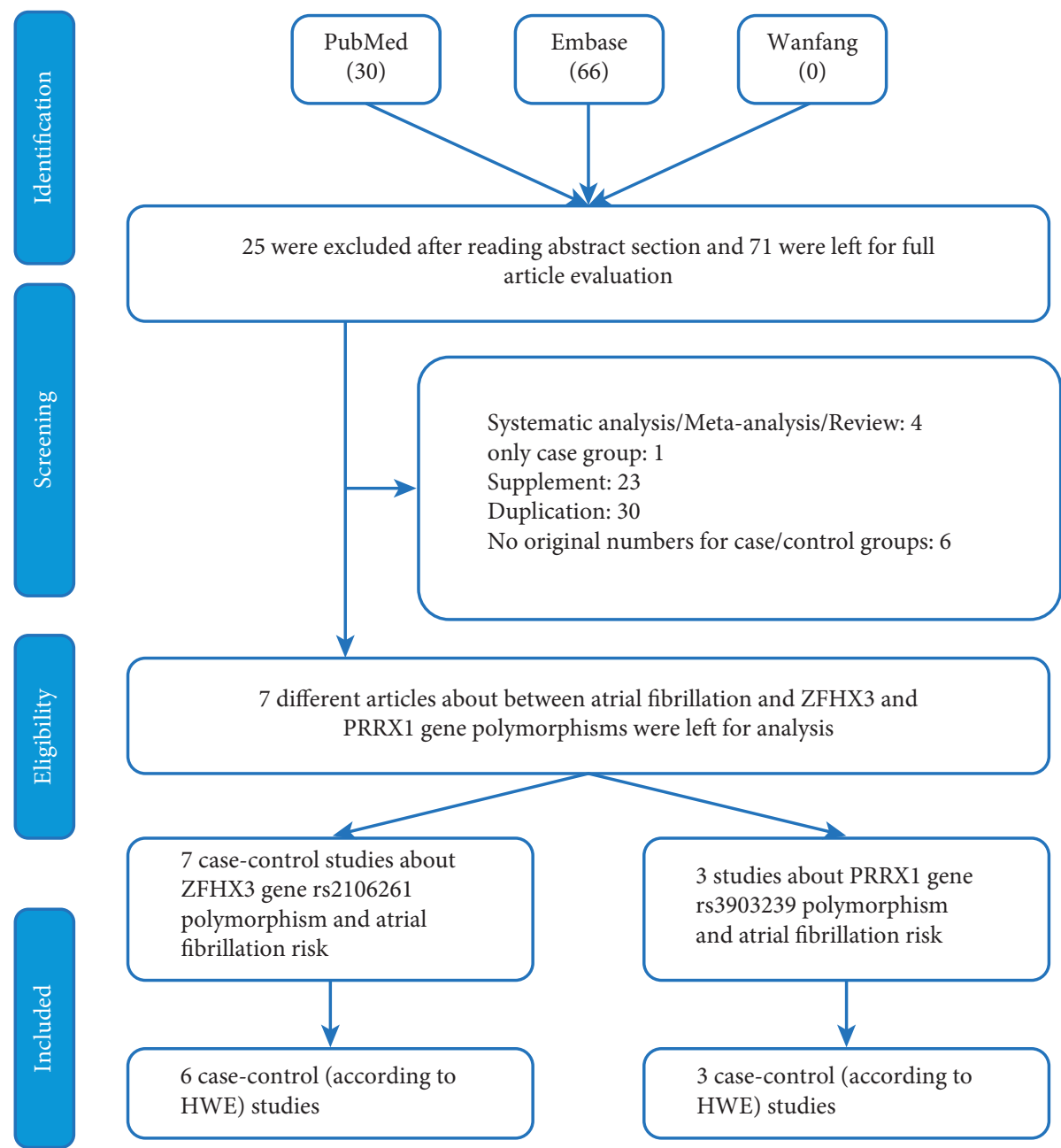

FIGURE 1: A flowchart showing the search strategy applied to search the related papers for ZFHX3 rs2106261 and PRRX1 rs3903239 polymorphisms and AF risk.

TABLE 1: Characteristics of studies of ZFHX3 and PRRX1 genes' two common polymorphisms and atrial fibrillation risk included in our meta-analysis.

\begin{tabular}{|c|c|c|c|c|c|c|c|c|c|c|c|c|c|c|c|c|}
\hline \multirow{2}{*}{$\begin{array}{l}\text { Author } \\
\text { ZFHX3 } \\
\text { rs2106261 }\end{array}$} & \multirow[b]{2}{*}{ Year } & \multirow[b]{2}{*}{ Country } & \multirow[b]{2}{*}{ Ethnicity } & \multirow[b]{2}{*}{ Case } & \multirow[b]{2}{*}{ Control } & \multicolumn{3}{|c|}{ Case } & \multicolumn{3}{|c|}{ Control } & \multirow[b]{2}{*}{ SOC } & \multirow[b]{2}{*}{ HWE } & \multirow[b]{2}{*}{ Genotype } & \multirow[b]{2}{*}{ NOS } & \multirow[b]{2}{*}{ AF type } \\
\hline & & & & & & $\mathrm{AA}$ & $\mathrm{AG}$ & GG & AA & AG & GG & & & & & \\
\hline Okubo & 2020 & Japan & Asian & 289 & 287 & 46 & 143 & 99 & 32 & 109 & 146 & $\mathrm{HB}$ & 0.096 & TaqMan & 8 & NA \\
\hline Zaw & 2017 & Japan & Asian & 411 & 1765 & 54 & 182 & 175 & 151 & 725 & 889 & $\mathrm{HB}$ & 0.853 & Illumina & 8 & NA \\
\hline Huang & 2015 & China & Asian & 569 & 1996 & 99 & 237 & 233 & 216 & 869 & 911 & $\mathrm{~PB}$ & 0.683 & HRM & 9 & $\mathrm{~A}$ \\
\hline Huang & 2015 & China & Asian & 641 & 1692 & 103 & 279 & 259 & 197 & 707 & 788 & $\mathrm{~PB}$ & 0.048 & HRM & 9 & A \\
\hline Huang & 2015 & China & Asian & 810 & 1627 & 128 & 369 & 313 & 149 & 726 & 752 & $\mathrm{~PB}$ & 0.163 & HRM & 9 & A \\
\hline Liu & 2014 & China & Asian & 593 & 996 & 110 & 299 & 184 & 99 & 446 & 451 & $\mathrm{HB}$ & 0.460 & MassARRAY & 8 & $\begin{array}{c}\text { Paroxysmal } \\
\text { AF }\end{array}$ \\
\hline Tomomori & 2018 & Japan & Asian & 362 & 627 & 50 & 181 & 131 & 60 & 250 & 317 & $\mathrm{HB}$ & 0.298 & TaqMan & 8 & $\begin{array}{c}\text { Paroxysmal } \\
\text { AF }\end{array}$ \\
\hline $\begin{array}{l}\text { PRRX1 } \\
\text { rs3903239 }\end{array}$ & & & & & & $\mathrm{CC}$ & $\mathrm{CT}$ & $\mathrm{TT}$ & $\mathrm{CC}$ & CT & $\mathrm{TT}$ & & & & & \\
\hline Kalinderi & 2018 & Greece & European & 167 & 12 & 15 & 62 & 90 & 8 & 49 & 67 & $\mathrm{~PB}$ & 0.809 & RCR-RFLP & 7 & NA \\
\hline Okubo & 2020 & Japan & Asian & 287 & 287 & 29 & 139 & 119 & 59 & 143 & 85 & $\mathrm{HB}$ & 0.935 & TaqMan & 8 & NA \\
\hline Liu & 2015 & China & Asian & 591 & 996 & 79 & 263 & 249 & 155 & 463 & 378 & $\mathrm{HB}$ & 0.503 & MassARRAY & 8 & Mixed \\
\hline
\end{tabular}

HB: hospital based; PB: population based; SOC; source of control; PCR-RFLP: polymerase chain reaction followed by restriction fragment length polymorphism; HRM: high-resolution melt; HWE: Hardy-Weinberg equilibrium of the control group; NA: not available; NOS: Newcastle-Ottawa Scale. 


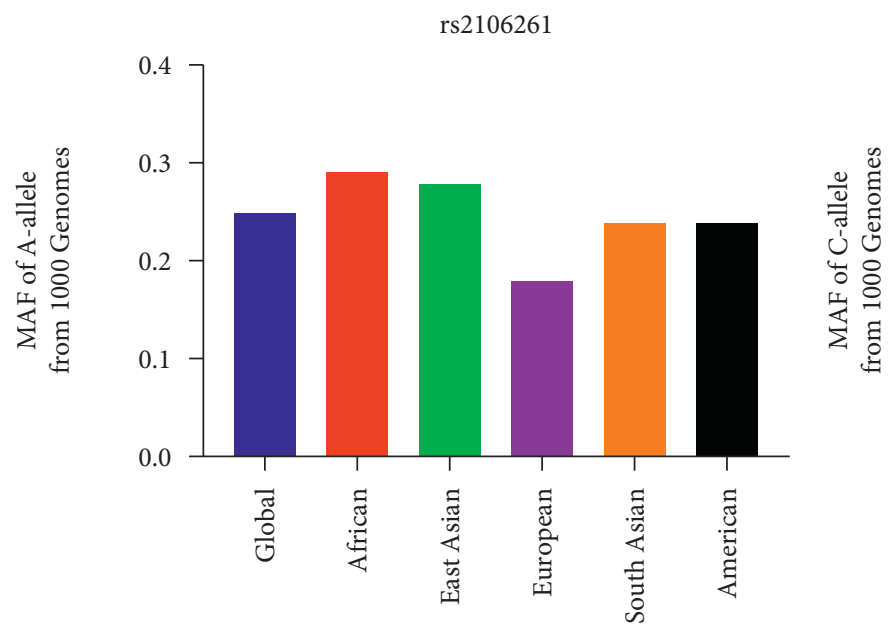

Ethnicity

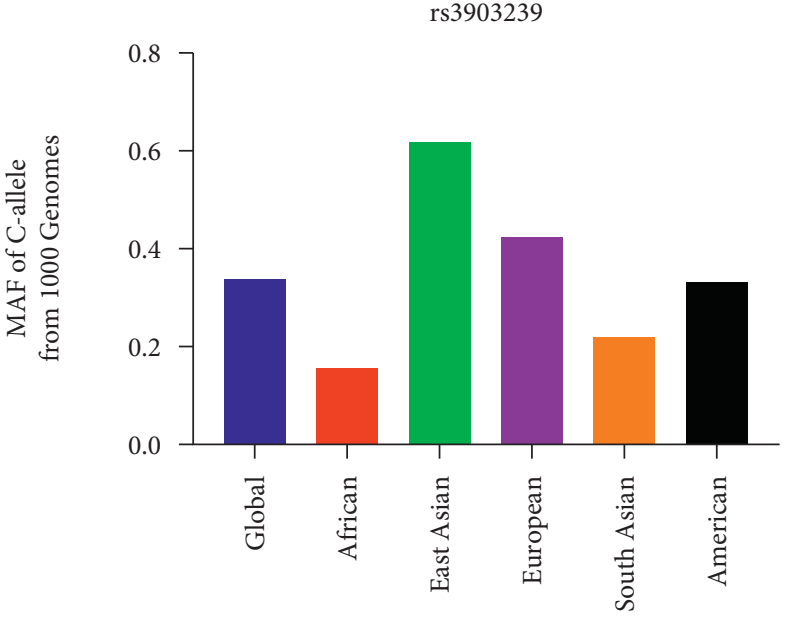

Ethnicity

(a)

(b)

FIgURE 2: MAF for the gene polymorphisms among different ethnicities. Vertical line, MAF; horizontal line, ethnicity type. EAS: East Asian; EUR: European; AFR: African; AMR: American; and SAS: South Asian. (a) rs2106261 and (b) rs3903239.

Figure 3(c)), the dominant model (OR $[95 \% \mathrm{CI}]=1.49$ $[1.30-1.70], P_{\text {heterogeneity }}=0.011, P<0.001$, Figure $\left.3(\mathrm{~d})\right)$, and $\mathrm{AA} \quad v s . \quad \mathrm{AC}+\mathrm{CC} \quad(\mathrm{OR} \quad[95 \% \quad \mathrm{CI}]=1.70 \quad[1.52-1.90]$, $P_{\text {heterogeneity }}=0.643, P<0.001$, Figure $3(\mathrm{e})$ ) (Table 2).

In the subgroup analysis by source of control, the ZFHX3 rs2106261 A-allele or AA genotype acted as a risk factor in both $\mathrm{HB}$ and $\mathrm{PB}$ subgroups: $\mathrm{HB}$ (such as A-allele vs. C-allele: OR $[95 \% \mathrm{CI}]=1.51 \quad[1.38-1.64], \quad P_{\text {(heterogeneity) }}=0.302$, $P<0.001 ;$ AC vs. CC: OR [95\% CI] $=1.57$ [1.38-1.79], $P_{\text {(heterogeneity) }}=0.156, P<0.001$ ), and $\mathrm{PB}$ (such as: A-allele $v s$. C-allele: $\quad$ OR $\quad[95 \% \quad \mathrm{CI}]=1.31 \quad[1.21-1.41]$, $P_{\text {(heterogeneity) }}=0.321, P<0.001 ;$ AC vs. CC: OR $[95 \% \mathrm{CI}]=$ $1.17 \quad[1.04-1.30], \quad P_{\text {(heterogeneity) }}=0.584, \quad P=0.007$ ) (Figures 3(a) and 3(b), Table 2).

To detect whether an association exists between genotype methods and the ZFHX3 rs2106261 polymorphism, we performed the next step. Several positive results were found in TaqMan (in the allelic contrast $(\mathrm{OR}=1.55,95 \%$ $\mathrm{CI}=1.33-1.80, P=0.740$ for heterogeneity, $P<0.001$ for significance), the heterozygote comparison ( $O R=1.82,95 \%$ $\mathrm{CI}=1.46-2.27, P=0.668$ for heterogeneity, $P<0.001)$, AA vs. $\mathrm{CC}\left(\mathrm{OR}=2.06,95 \% \mathrm{CI}=1.48-2.86, P_{\text {heterogeneity }}=0.884\right.$, $P<0.001$ for significance), the dominant model (OR $[95 \%$ $\left.\mathrm{CI}]=1.87[1.52-2.30], P_{\text {heterogeneity }}=0.674, P<0.001\right)$, and $\mathrm{AA} \quad v s . \quad \mathrm{AC}+\mathrm{CC} \quad(\mathrm{OR} \quad[95 \% \quad \mathrm{CI}]=1.51 \quad[1.11-2.06]$, $P_{\text {heterogeneity }}=1.000, P<0.001$ ), high-resolution melt (in the allelic contrast $(\mathrm{OR}=1.31, \quad 95 \% \quad \mathrm{CI}=1.21-1.41$, $\left.P_{\text {heterogeneity }}=0.647, P<0.001\right)$, the heterozygote comparison $(\mathrm{OR}=1.17,95 \% \mathrm{CI}=1.04-1.30, P=0.584$ for heterogeneity, $P=0.007$ for significance), AA vs. CC $(\mathrm{OR}=1.81$, 95\% CI $\left.=1.54-2.12, \quad P_{\text {heterogeneity }}=0.417, \quad P<0.001\right)$, the dominant model $(\mathrm{OR}=1.29,95 \% \mathrm{CI}=1.16-1.43, P=0.655$ for heterogeneity, $P<0.001)$, and $\mathrm{AA} v s$. $\mathrm{AC}+\mathrm{CC}$
$\left(\mathrm{OR}=1.68, \quad 95 \% \quad \mathrm{CI}=1.45-1.94, \quad P_{\text {heterogeneity }}=0.384\right.$, $P<0.001$ for significance), and others (data not shown)) (Figure 4 and Table 2).

3.3. PRRX1 rs3903239 and AF Risk. Decreased associations were found in the heterozygote comparison (OR $[95 \% \mathrm{CI}]=$ 0.83 [0.77-0.99], $P_{\text {heterogeneity }}=0.522, P=0.036$, Figure 5(a) and Table 2) and dominant model (OR $[95 \% \mathrm{CI}]=0.79$ [0.67-0.94], $P=0.137$ for heterogeneity, $P=0.006$, Figure 5(b) and Table 2).

3.4. Sensitivity Analysis and Publication Bias. Begg's funnel chart and Egger's test were performed to assess publication bias. The results did not show any evidence of publication bias (for example, A-allele vs. G-allele, $t=1.46, P=0.205$ (Egger's test); $z=1.2, P=0.23$ (Begg's test) for ZFHX3 rs2106261, Figure 6; C-allele vs. T-allele, $t=0.11, P=0.933$ (Egger's test); $z=0.0, P=1.00$ (Begg's test) for PRRX1 rs3903239, Figure 7 and Table 3). Sensitivity analysis was performed to assess the impact of each individual study on the combined OR by removing individual studies sequentially. The results suggested that no separate study significantly affected the overall OR for ZFHX3 rs2106261 (Figure 8).

3.5. ZFHX3 and PRRX1 Interaction Networks. A network of potential gene-gene interactions for ZFHX3 and PRRX1 genes was analyzed using the String online web page (https:// string-db.org/) [33] (Figure 9). Each gene showed ten significantly related genes. 


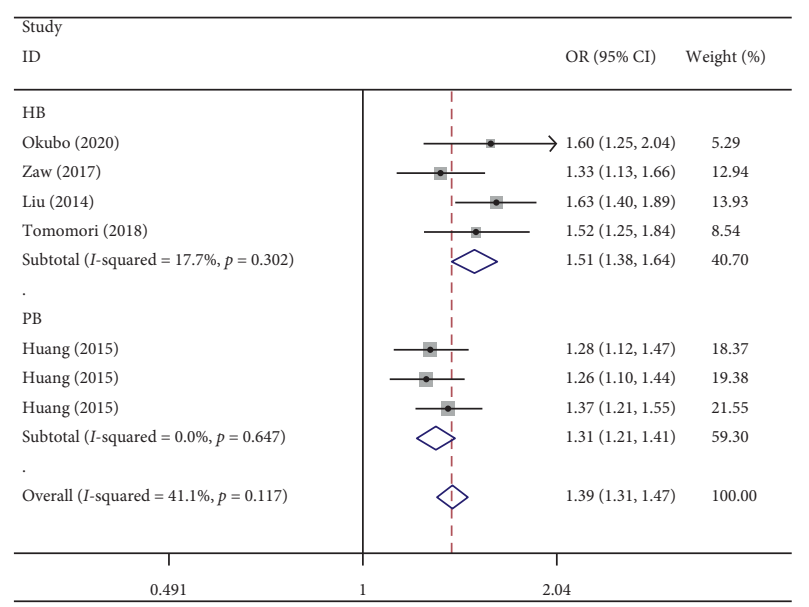

(a)

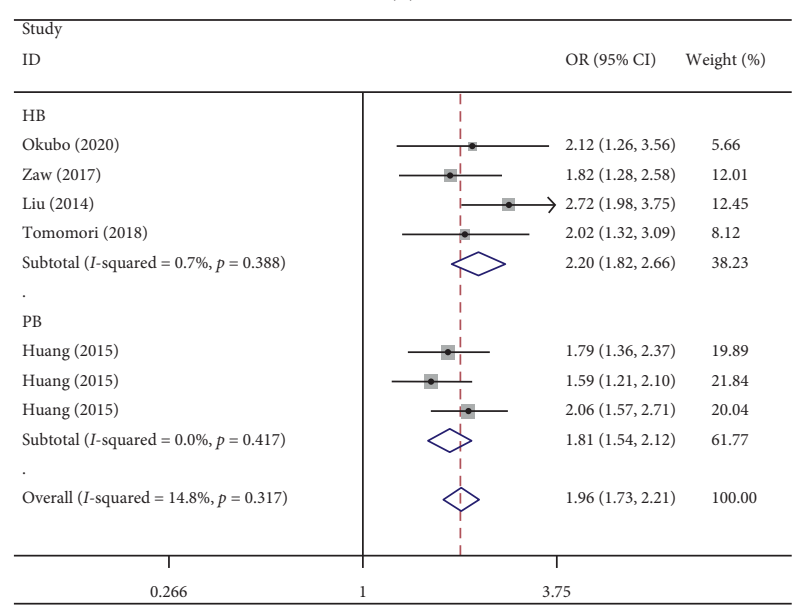

(c)

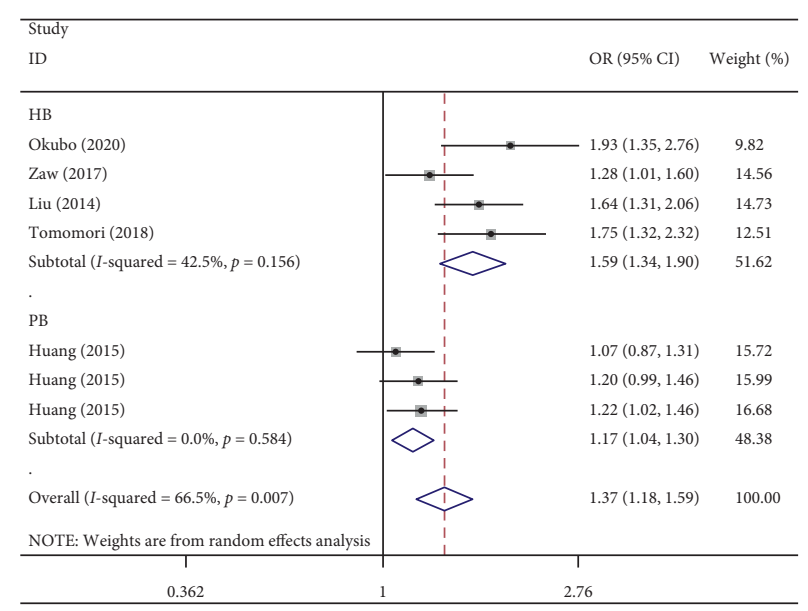

(b)

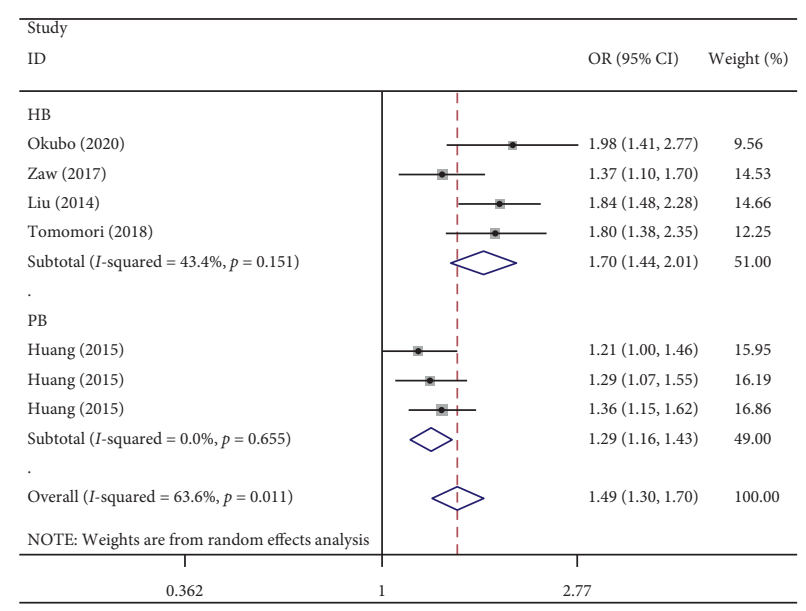

(d)

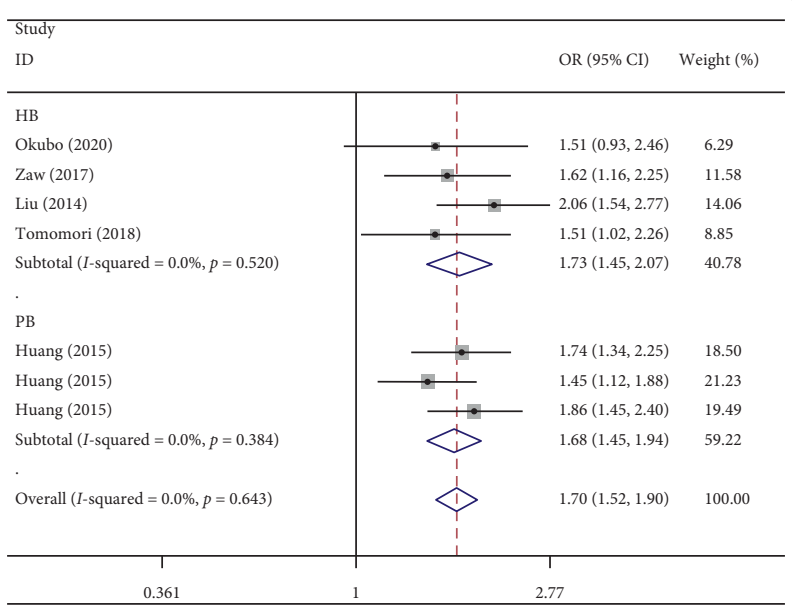

(e)

FIGURE 3: Forest plot of AF risk associated with ZFHX3 rs2106261 polymorphism in all genetic models by source of the control subgroup. The squares and horizontal lines correspond to the study-specific OR and 95\% CI. The area of the squares reflects the weight (inverse of the variance). The diamond represents the summary OR and 95\% CI. (a) A-allele vs. C-allele; (b) AC vs. CC; (c) AA vs. CC; (d) AA + AC vs. CC; and (e) AA vs. AC+CC. 
TABLE 2: Stratified analyses of ZFHX3 and PRRX1 genes' two common polymorphisms on atrial fibrillation risk.

\begin{tabular}{|c|c|c|c|c|c|c|c|}
\hline $\begin{array}{l}\text { Variables } \\
\text { ZFHX3 } \\
\text { rs2106261 } \\
\end{array}$ & $N$ & $\begin{array}{l}\text { Case/ } \\
\text { Control }\end{array}$ & $\begin{array}{l}\text { M-allele vs. W-allele } \\
\text { OR(95\%CI) } P_{h} P I^{2}\end{array}$ & $\begin{array}{c}\text { MW vs. WW } \\
\text { OR(95\%CI) } P_{h} P I^{2}\end{array}$ & $\begin{array}{l}\mathrm{MM}+\mathrm{MW} \text { vs. } \mathrm{WW} \\
\mathrm{OR}(95 \% \mathrm{CI}) P_{h} P I^{2}\end{array}$ & $\begin{array}{c}\text { MM vs. WW } \\
\text { OR(95\%CI) } P_{h} P I^{2}\end{array}$ & $\begin{array}{l}\text { MM vs. } \mathrm{MW}+\mathrm{WW} \\
\mathrm{OR}(95 \% \mathrm{CI}) P_{h} P I^{2}\end{array}$ \\
\hline Total & 7 & $4 /$ & $\begin{array}{r}1.39(1.3 \\
0.00 \\
\end{array}$ & $\begin{array}{r}1.37(1.1 \\
0.0 \\
\end{array}$ & $\begin{array}{l}0.011 \\
6 \\
\end{array}$ & $\begin{array}{r}1.96(1.7 \\
0.0 \\
\end{array}$ & $\begin{array}{r}1 . \\
0.6 \\
\end{array}$ \\
\hline \multicolumn{8}{|l|}{ SOC } \\
\hline $\mathrm{HB}$ & 4 & $\begin{array}{l}1654 / \\
3675\end{array}$ & $\begin{array}{c}1.51(1.38- \\
0.000\end{array}$ & $\begin{array}{r}1.57(1.38- \\
0.000\end{array}$ & $\begin{array}{r}1.68(1.49-1 . \\
0.00043\end{array}$ & $\begin{array}{r}2.20( \\
0.388\end{array}$ & $\begin{array}{c}1.73(1.45-2.07) \\
0.520 \quad 0.000 \quad 0.0 \%\end{array}$ \\
\hline $\mathrm{PB}$ & 3 & $\begin{array}{c}2020 / \\
5315\end{array}$ & $\begin{array}{c}1.31(1.21-1.41) 0.321 \\
0.0000 .0 \%\end{array}$ & $\begin{array}{c}1.17(1.04-1.30) 0.584 \\
0.0070 .0 \%\end{array}$ & $\begin{array}{c}1.29(1.16-1.43) 0.655 \\
0.0000 .0 \%\end{array}$ & $\begin{array}{c}1.81(1.54-2.12) 0.417 \\
0.0000 .0 \%\end{array}$ & $\begin{array}{c}1.68(1.45-1.94) \\
0.3840 .0000 .0 \%\end{array}$ \\
\hline \multicolumn{8}{|l|}{ Genotype } \\
\hline Taq & 2 & $650 / 914$ & $\begin{array}{c}1.55(1.33-1.80) \\
0.740 \quad 0.000 \quad 0.0 \%\end{array}$ & $\begin{array}{c}1.82(1.46-2.27) \\
0.6680 .000 \quad 0.0 \%\end{array}$ & & $\begin{array}{c}2.06(1.48-2.86) \\
0.8840 .000 \quad 0.0 \%\end{array}$ & $\begin{array}{c}1.51(1.11-2.06) \\
1.0000 .0000 .0 \%\end{array}$ \\
\hline Other & 2 & $\begin{array}{l}1004 / \\
2761\end{array}$ & $\begin{array}{c}1.47(1.21-1.80) \\
0.0680 .00070 .1 \%\end{array}$ & $\begin{array}{c}1.45(1.24-1.70) 0.123 \\
0.00058 .1 \%\end{array}$ & $\begin{array}{c}1.59(1.19-2.12) 0.057 \\
0.00272 .4 \%\end{array}$ & $\begin{array}{c}1.47(1.21-1.80) 0.095 \\
0.00064 .1 \%\end{array}$ & $\begin{array}{c}1.86(1.50-2.32) \\
0.2790 .00014 .5 \%\end{array}$ \\
\hline HRM & 3 & $\begin{array}{c}2020 / \\
5315\end{array}$ & $\begin{array}{c}1.31(1.21-1.41) 0.647 \\
0.0000 .0 \%\end{array}$ & $\begin{array}{c}1.17(1.04-1.30) 0.584 \\
0.0070 .0 \% \\
\end{array}$ & $\begin{array}{c}1.29(1.16-1.43) 0.655 \\
0.0000 .0 \%\end{array}$ & $\begin{array}{c}1.81(1.54-2.12) 0.417 \\
0.0000 .0 \% \\
\end{array}$ & $\begin{array}{l}1.94) \\
0.4 \%\end{array}$ \\
\hline \multicolumn{8}{|c|}{ PRRX1 rs3903239 } \\
\hline Total & 3 & $\begin{array}{l}1045 / \\
1407\end{array}$ & $\begin{array}{c}0.82(0.63-1.07) \\
0.0230 .14773 .5 \% \\
\end{array}$ & $\begin{array}{c}0.83(0.77-0.99) \\
0.5220 .0360 .0 \% \\
\end{array}$ & $\begin{array}{c}0.79(0.67-0.94) \\
0.1370 .00649 .7 \% \\
\end{array}$ & $\begin{array}{c}0.68(0.35-1.32) \\
0.0110 .25378 .0 \% \\
\end{array}$ & $\begin{array}{c}0.75(0.42-1.31) \\
0.0230 .31073 .5 \% \\
\end{array}$ \\
\hline
\end{tabular}

$P_{h}$ : value of the $Q$-test for the heterogeneity test; $P$ : $Z$-test for the statistical significance of the OR.

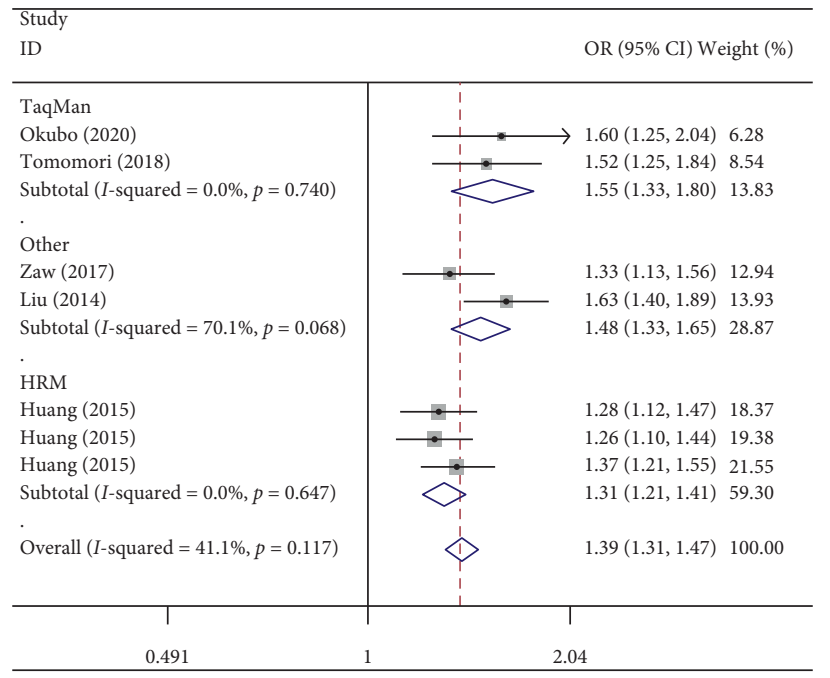

(a)

\begin{tabular}{llll}
\hline Study & OR $(95 \%$ CI) Weight (\%) \\
ID & & & \\
TaqMan \\
Okubo (2020) \\
Tomomori (2018) \\
Subtotal $(I$-squared $=0.0 \%, p=0.888)$ \\
Other
\end{tabular}

(c)

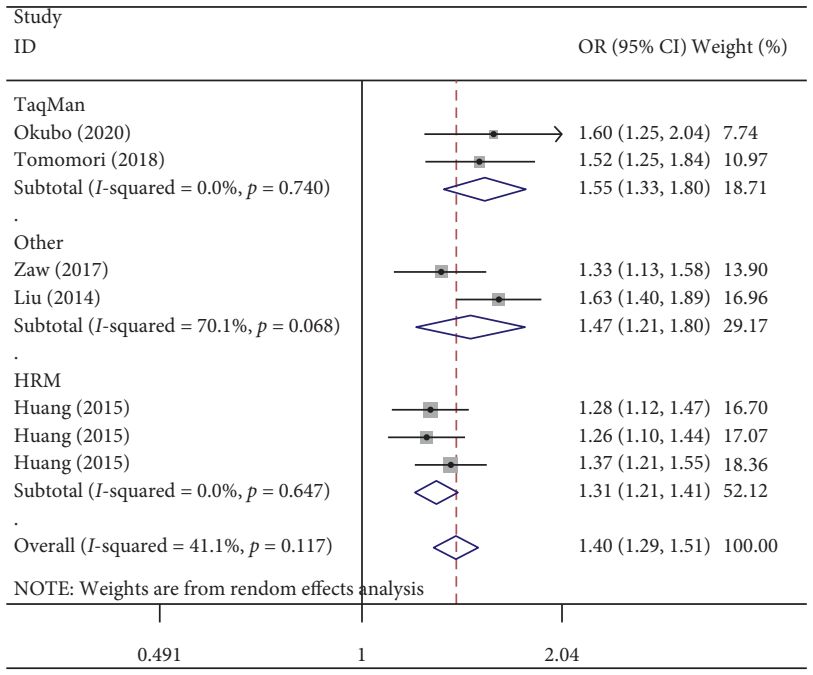

(b)

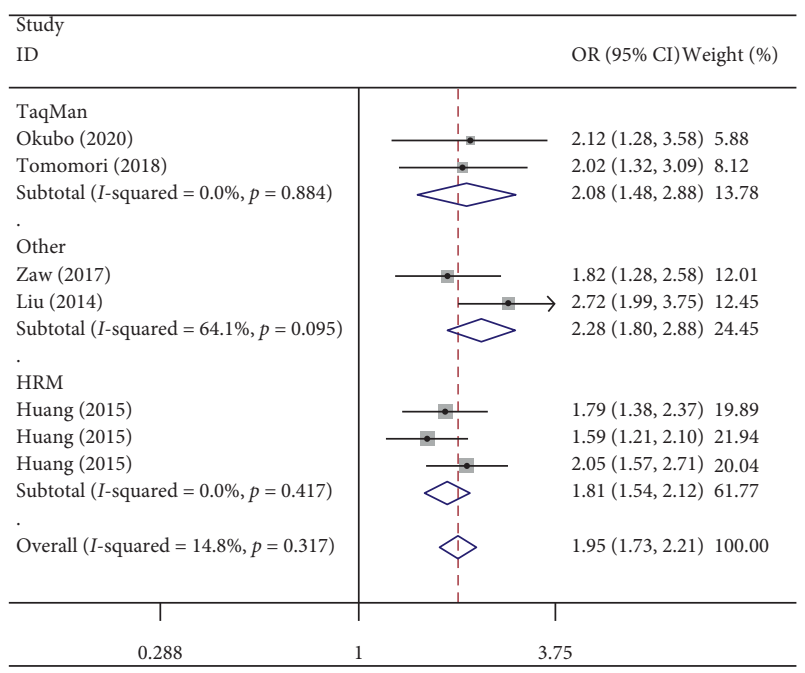

(d)

Figure 4: Continued. 


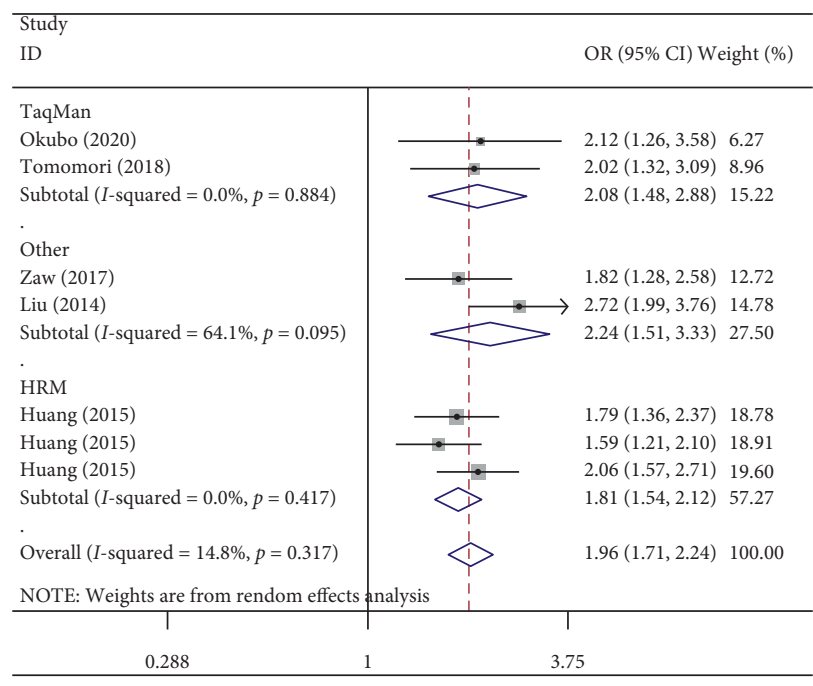

(e)

\begin{tabular}{|c|c|c|c|}
\hline $\begin{array}{l}\text { Study } \\
\text { ID }\end{array}$ & & $\mathrm{OR}(95 \% \mathrm{CI}) \mathrm{We}$ & ight (\%) \\
\hline TaqMan & & & \\
\hline Okubo (2020) & & $1.98(1.41,2.77)$ & 9.58 \\
\hline Tomomori (2018) & & $1.80(1.38,2.35)$ & 12.25 \\
\hline Subtotal $(I$-squared $=0.0 \%, p=0.874)$ & & $1.87(1.52,2.30)$ & 21.81 \\
\hline Other & & & \\
\hline Zaw (2017) & $\longrightarrow$ & $1.37(1.10,1.70)$ & 14.53 \\
\hline Liu (2014) & - & $1.04(1.40,2.28)$ & 14.66 \\
\hline Subtotal $(I-$ squared $=72.4 \%, p=0.067)$ & & $1.69(1.19,2.12)$ & 29.19 \\
\hline HRM & & & \\
\hline Huang (2015) & $=$ & $1.21(1.00,1.46)$ & 16.96 \\
\hline Huang (2015) & $\longrightarrow$ & $1.29(1.07,1.55)$ & 16.19 \\
\hline Huang (2015) & $\rightarrow$ & $1.36(1.15,1.52)$ & 16.96 \\
\hline Subtotal $(I$-squared $=0.0 \%, p=0.556)$ & $\diamond$ & $1.29(1.15,1.43)$ & 49.00 \\
\hline Overall $(I$-squared $=63.6 \%, p=0.011)$ & & $1.49(1.30,1.70)$ & 100.00 \\
\hline NOTE: Weights are from random effect & Analysis & & \\
\hline 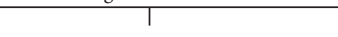 & & & \\
\hline 0.352 & 1 & 77 & \\
\hline
\end{tabular}

(g)

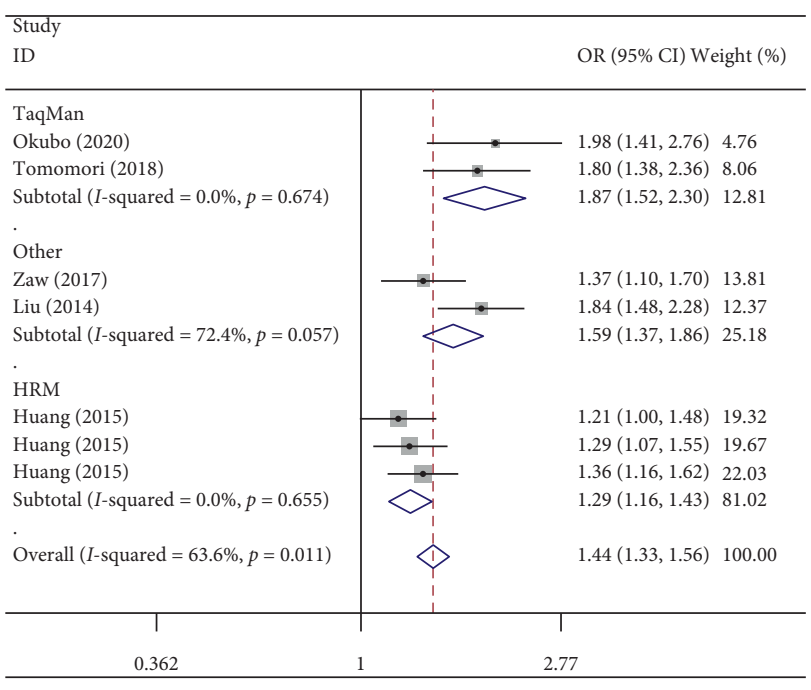

(f)

\begin{tabular}{lllll}
\hline Study \\
ID
\end{tabular}

(h)

Figure 4: Forest plot of AF risk associated with ZFHX3 rs2106261 polymorphism in the genotype method subgroup. (a) A-allele vs. C-allele (fixed-model); (b) A-allele vs. C-allele (random-model); (c) AC vs. CC; (d) AA + AC vs. CC (fixed-model); (e) AA + AC vs. CC (randommodel); (f) AA vs. CC (fixed-model); (g) AA vs. CC (random-model); and (h) AA vs. AC + CC.

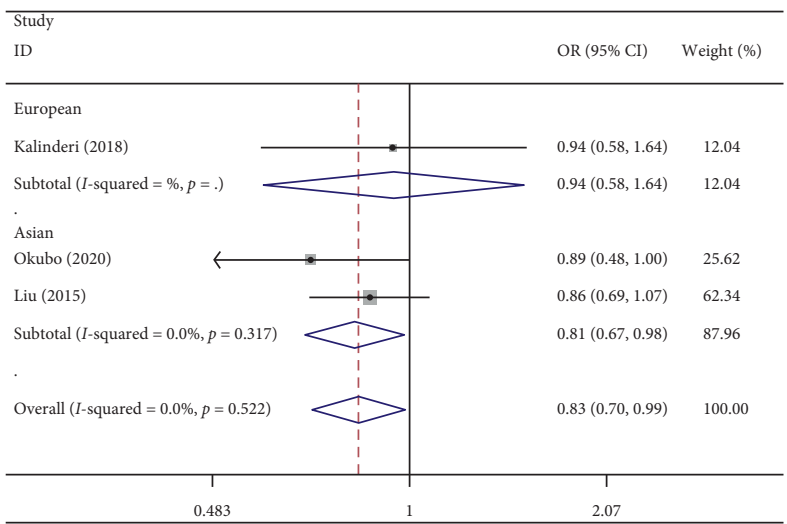

(a)

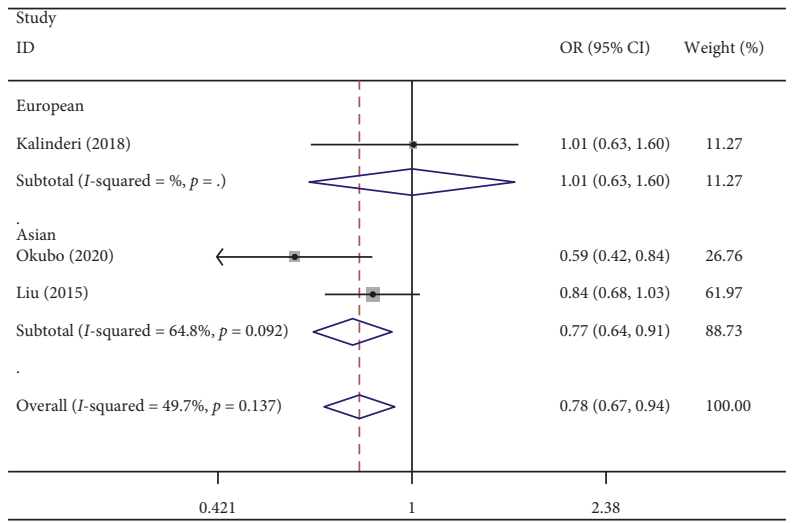

(b)

FIGURE 5: Forest plot of AF risk associated with PRRX1 rs3903239 polymorphism in the whole analysis. (a) Heterozygote comparison; (b) dominant model. 


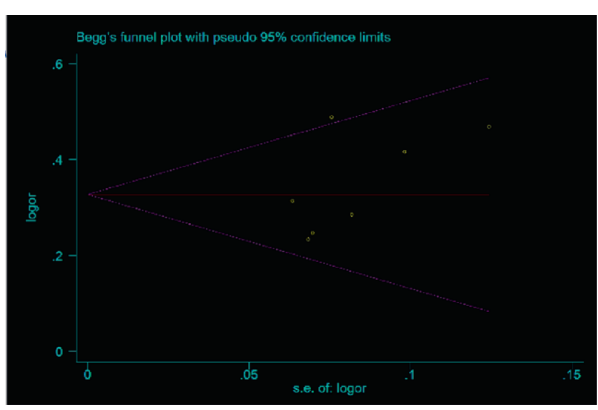

(a)

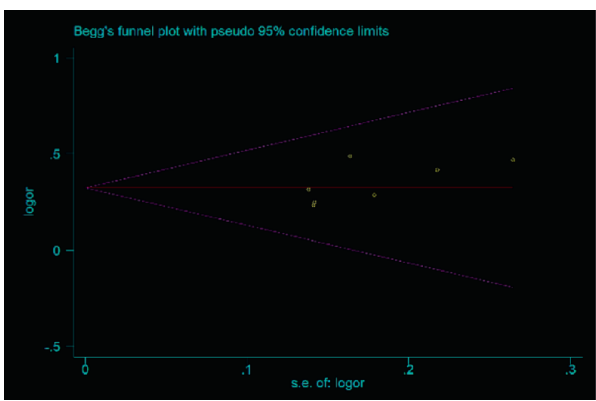

(c)

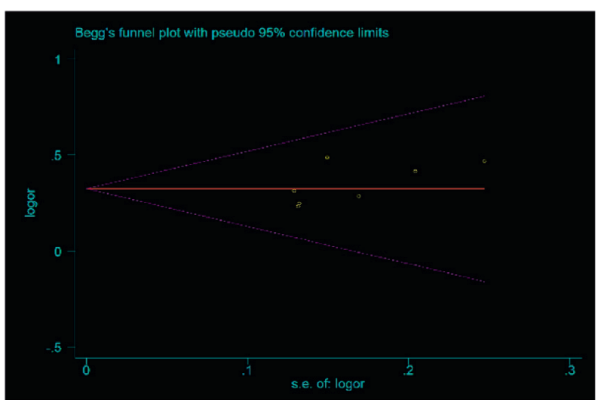

(e)

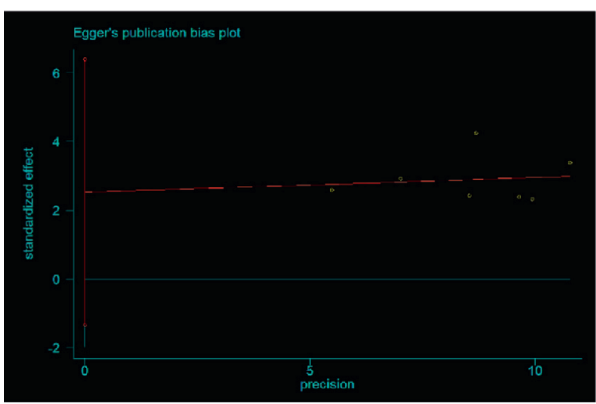

(g)

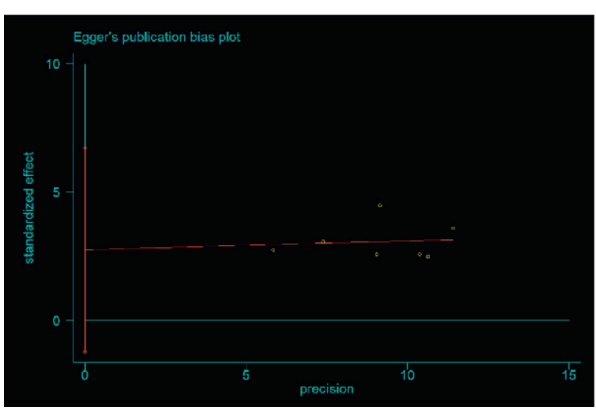

(i)

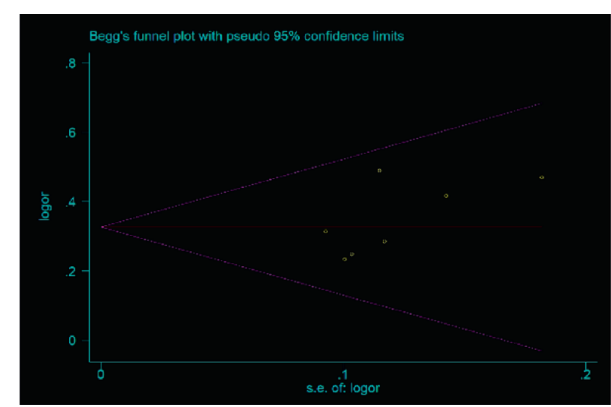

(b)

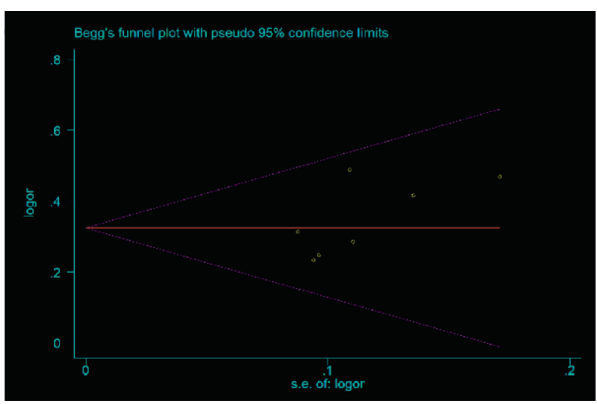

(d)

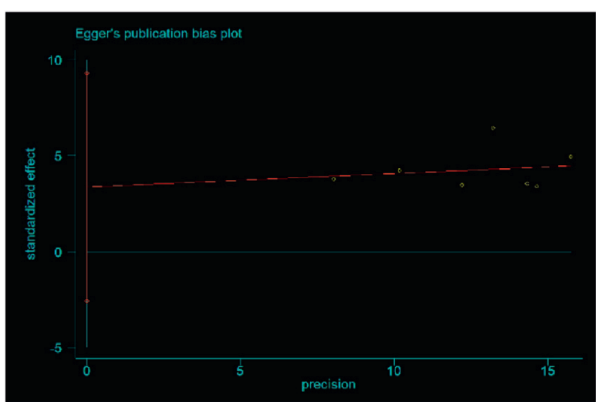

(f)

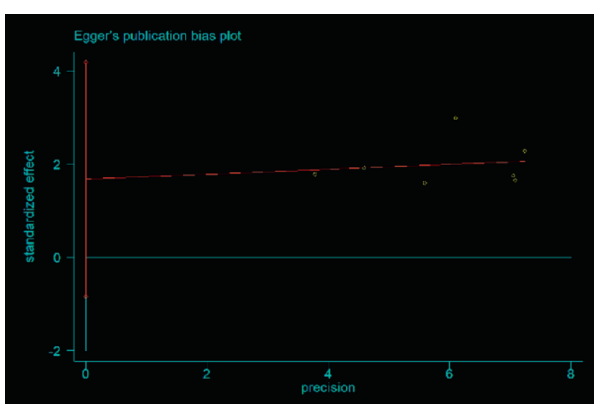

(h)

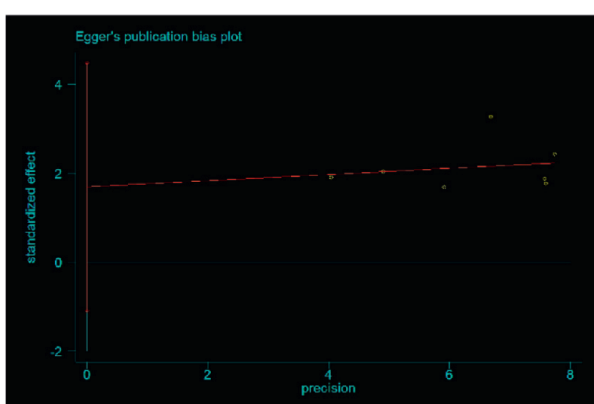

(j)

FIGURE 6: Begg's and Egger's tests for publication bias plot in all genetic models (ZFHX3 rs2106261 polymorphism). (a) A-allele vs. C-allele; (b) AC vs. CC; (c) AA vs. CC; (d) AA + AC vs. CC; (e) AA vs. AC + CC for Begg's test; (f) A-allele vs. C-allele; (g) AC vs. CC; (h) AA vs. CC; (i) $\mathrm{AA}+\mathrm{AC}$ vs. CC; and (j) AA vs. AC+ CC for Egger's test. 


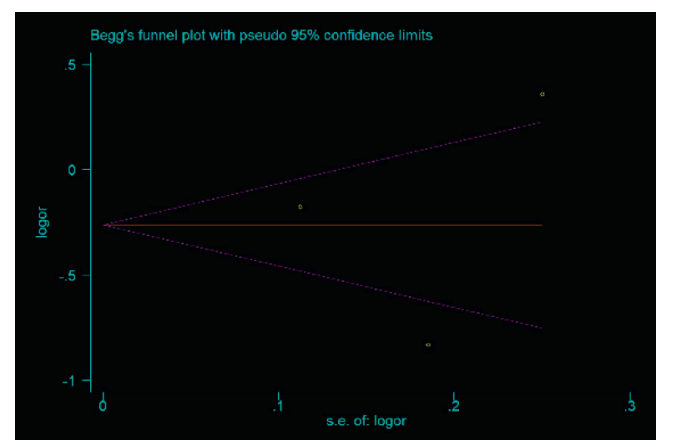

(a)

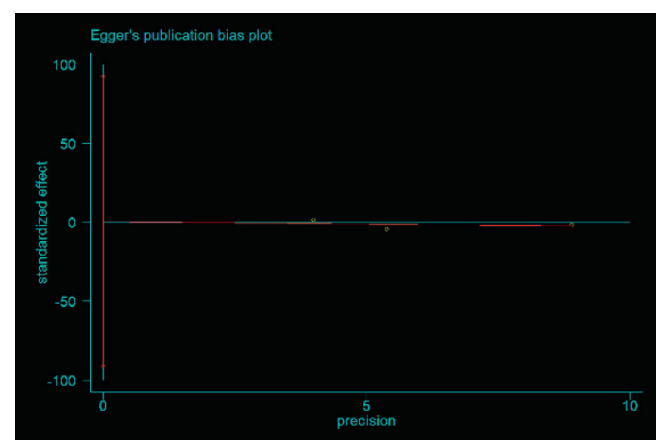

(c)

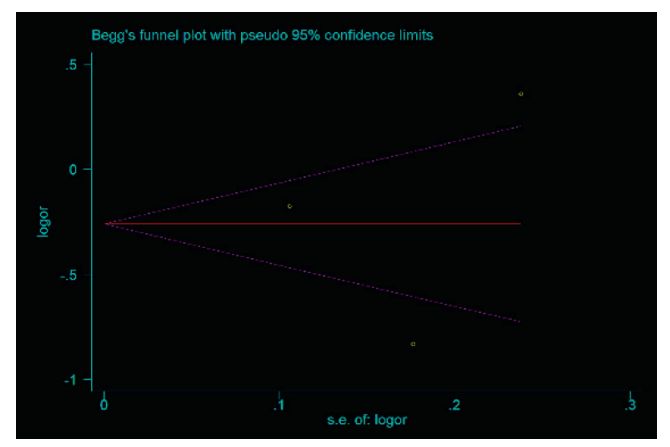

(b)

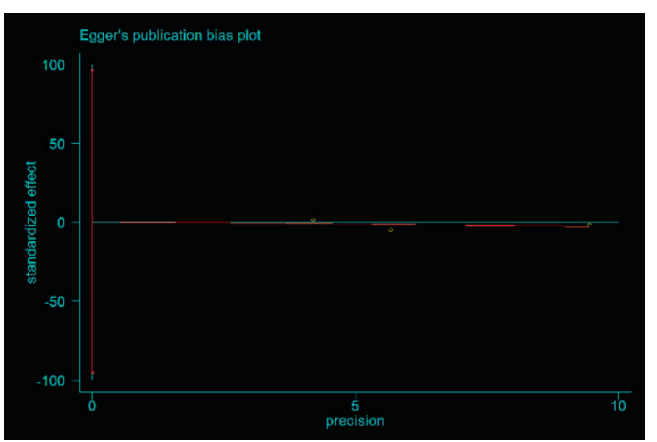

(d)

FIGURE 7: Begg's and (c, d) Egger's tests for publication bias plot in the two models (PRRX1 rs3903239 polymorphism): heterozygote comparison and dominant model.

TABle 3: Publication bias tests (Begg's funnel plot and Egger's test for the publication bias test) for ZFHX3 and PRRX1 genes' two common polymorphisms (rs2106261 and rs3903239).

\begin{tabular}{|c|c|c|c|c|c|c|c|}
\hline \multirow{2}{*}{$\begin{array}{l}\text { Egger's test } \\
\text { Genetic type }\end{array}$} & \multirow[b]{2}{*}{ Coefficient } & \multirow[b]{2}{*}{ Standard error } & \multirow[b]{2}{*}{$t$} & \multirow[b]{2}{*}{$P$ value } & \multirow[b]{2}{*}{$95 \% \mathrm{CI}$ of intercept } & \multicolumn{2}{|c|}{ Begg's test } \\
\hline & & & & & & $z$ & $P$ value \\
\hline \multicolumn{8}{|l|}{ ZFHX3 rs2106261 } \\
\hline A-allele vs. G-allele & 3.372 & 2.313 & 1.46 & 0.205 & $(-2.573-9.317)$ & 1.2 & 0.23 \\
\hline AG vs. GG & 2.523 & 1.507 & 1.67 & 0.155 & $(-1.351-6.398)$ & 1.2 & 0.23 \\
\hline $\mathrm{AA}+\mathrm{AG}$ vs. $\mathrm{GG}$ & 2.744 & 1.543 & 1.78 & 0.133 & $(-1.223-6.712)$ & 1.2 & 0.23 \\
\hline AA vs. GG & 1.671 & 0.977 & 1.71 & 0.148 & $(-0.840-4.182)$ & 1.2 & 0.23 \\
\hline AA vs. AG + GG & 1.690 & 1.083 & 1.56 & 0.179 & $(-1.094-4.475)$ & 1.2 & 0.23 \\
\hline \multicolumn{8}{|l|}{ PRRX1 rs3903239 } \\
\hline C-allele vs. T-allele & 1.034 & 9.771 & 0.11 & 0.933 & $(-123.117-125.186)$ & 0.0 & 1.00 \\
\hline CT vs. TT & 0.496 & 7.243 & 0.07 & 0.956 & $(-91.538-92.531)$ & 0.0 & 1.00 \\
\hline $\mathrm{CC}+\mathrm{CT}$ vs. TT & 0.471 & 7.530 & 0.06 & 0.960 & $(-95.213-96.154)$ & 0.0 & 1.00 \\
\hline CC vs. TT & 0.251 & 3.834 & 0.07 & 0.958 & $(-48.468-48.971)$ & 0.0 & 1.00 \\
\hline $\mathrm{CC}$ vs. $\mathrm{CT}+\mathrm{TT}$ & 0.290 & 4.031 & 0.07 & 0.954 & $(-50.938-51.519)$ & 0.0 & 1.00 \\
\hline
\end{tabular}

\section{Discussion}

AF is considered to be the most common supraventricular arrhythmia, affecting up to $1 \%$ of the natural population $[34,35]$. With increasing age, the prevalence rate increases year by year, and the incidence of elderly cases ( $\geq 80$ years) can reach $8 \%$ [36]. Many types of heart and medical diseases that increase the risk of AF include arterial hypertension, cardiomyopathies, obstructive sleep apnea, and valve dysfunction [37, 38]. In addition, based on a recent metaanalysis of GWAS for AF [11], more than 100 AF risk genetic mutations and polymorphisms have been reported, indicating that gene polymorphisms are involved in the mechanisms of AF. An increasing number of studies have shown that genetic variation may promote the pathophysiology of AF by altering protein expression and function related to various cellular activities [39].

To date, several meta-analyses of gene polymorphisms and AF susceptibility have been published and have identified associations, including chromosome 4q25 variants, CYP11B2-344T $>$ C, and mink S38G [40-43]. A growing number of studies have identified polymorphisms in both ZFHX3 and PRRX1, and two previous meta-analyses have been involved with polymorphisms in the ZFHX3 gene, 


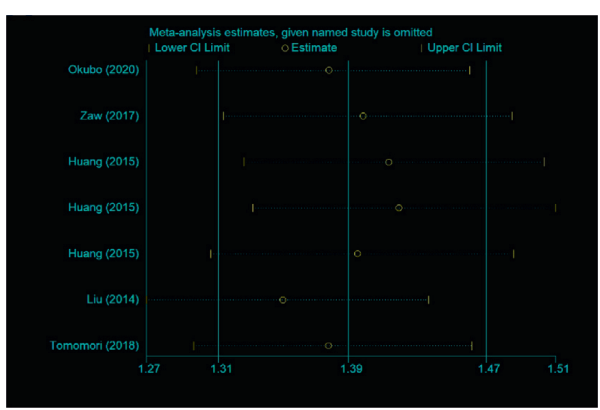

(a)

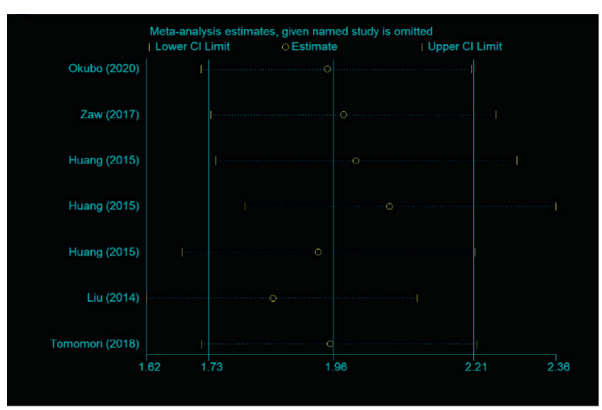

(c)

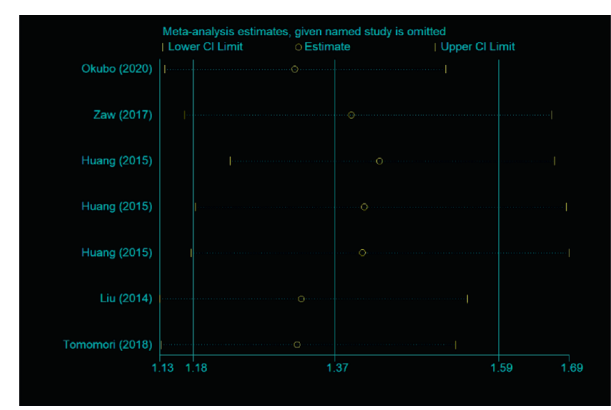

(b)

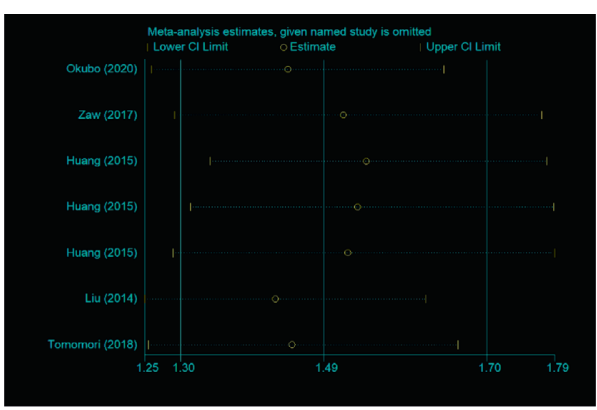

(d)

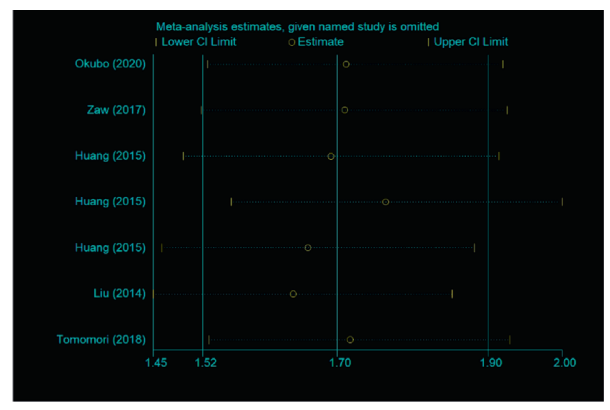

(e)

FIgURE 8: Sensitivity analysis between ZFHX3 rs2106261 polymorphism and AF risk (all five genetic models). (a) A-allele vs. C-allele; (b) AC vs. CC; (c) AA vs. CC; (d) AA + AC vs. CC; and (e) AA vs. AC+CC.

rather than the PRRX1 gene with AF susceptibility. Zhai et al. performed a meta-analysis of 10 case-control comparisons about rs7193343 polymorphism and found this polymorphism may be associated with risk of AF in the Caucasian population but not in the Asian population [44]. In addition, Jiang et al. also focused the polymorphisms for AF susceptibility through meta-analysis, and two polymorphisms in the ZFHX3 gene were analyzed (three studies about rs7193343 and only two studies about rs2106261), and no association was observed [45]. After that, other studies related to ZFHX3 gene rs2106261 polymorphism have been reported; moreover, another gene polymorphism (PRRX1 rs3903239) has been reported. Therefore, we aim to reanalyze the association between ZFHX3 rs2106261 or PRRX1 rs3903239 polymorphism and AF risk based on previous studies.

Previously, some relative studies have been reported. Zaw et al. showed that ZFHX3 rs2106261 polymorphism was a risk marker for AF and AF-related phenotypes [27]. Huang et al. performed large-population case-control studies. They found a significant A-allelic and genotypic association with $\mathrm{AF}$ in three different populations [22]. In addition, more highly significant associations were observed in the combined population. Liu et al. investigated a robust association between rs2106261 and increased risk of $\mathrm{AF}(\mathrm{OR}=1.71,95 \%$ $\left.\mathrm{CL}=1.46-2.00, P=1.85 \times 10^{-11}\right)$ [24]. However, Tomomori et al. found rs2106261 A-allele was associated with lower AF recurrence rate after pulmonary vein isolation, which was opposite to the other abovementioned studies [26]. On the other hand, Kalinderi et al. did not observe a positive association for PRRX1 rs3903239 polymorphism [18]. Okubo et al. identified five susceptible polymorphisms, including rs3903239 and rs2106261, and significant associations were demonstrated $\left(P=4.2 \times 10^{-5}\right.$ for rs3903239 and $3.87 \times 10^{-6}$ for rs2106261) [25]. Liu et al. confirmed that rs3903239 was a risk factor for $\mathrm{AF}(\mathrm{OR}=1.14,95 \% \mathrm{CI}=1.10-1.17)$.

The current analysis is to evaluate the associations between ZFHX3 rs2106261 or PRRX1 rs3903239 polymorphism and AF risk from a comprehensive analysis, involving 4719 cases and 10397 controls [24]. We found a relationship 


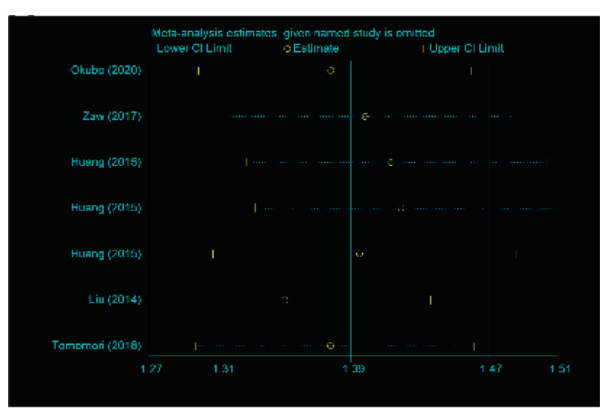

(a)

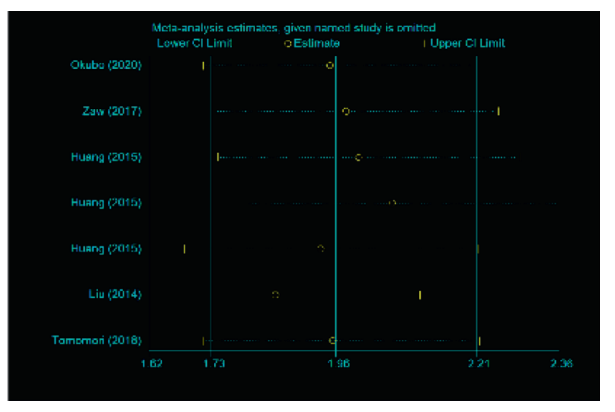

(c)

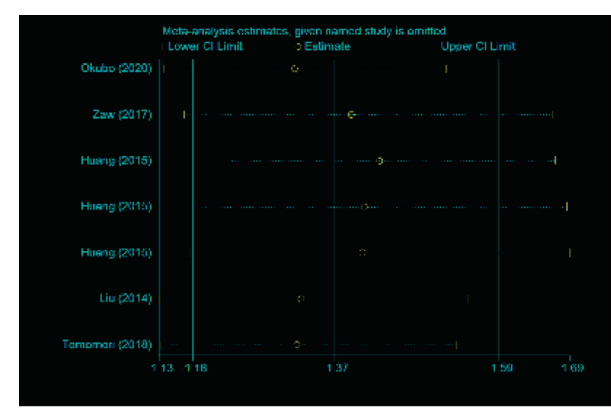

(b)

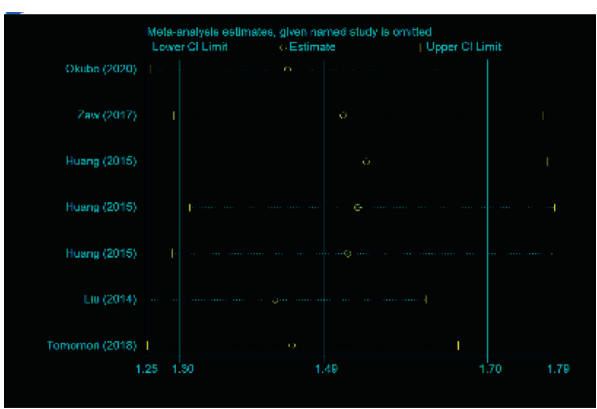

(d)

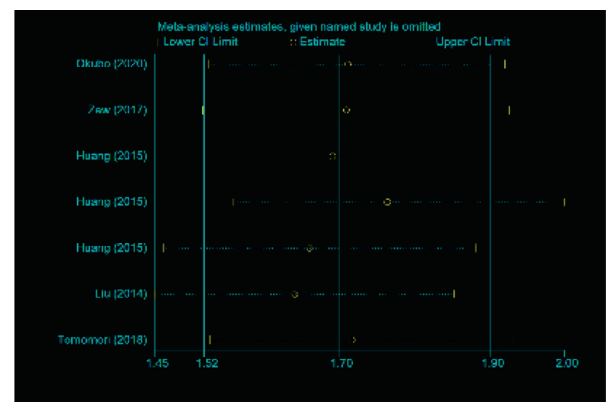

(e)

FIGURE 9: Human ZFHX3 and PRRX1 gene interactions network with other genes obtained from the String online server. At least, 10 genes have been indicated to correlate with the two abovementioned genes, respectively. (a, c) Network and ten related genes for the ZFHX3 gene; (b d) network and ten related genes for the PRRX1 gene.

between ZFHX3 rs2106261 and AF risk; in contrast, the PRRX1 rs3903239 polymorphism functioned as a protective factor in $\mathrm{AF}$ development. In other words, individuals carrying the A-allele of the ZFHX3 rs2106261 polymorphism may have a high risk of AF. Individuals with the CC or CT genotype of PRRX1 might have a decreased risk for AF. These findings can help reduce the incidence of AF through early detection and possible prevention measures. Different genes or polymorphisms in the same genes may play multiple roles in the progression of $\mathrm{AF}$, and this may explain the abovementioned conclusions.

In addition, the online analysis system String was applied to predict the potential functional partners of the genes, which may help to expand the range of vision of related genes. Ten genes were identified. The three highest scores of associations were for cyclin-dependent kinase inhibitor $1 \mathrm{~A}$ $(\mathrm{CDKN1A})($ score $=0.921)$, runt-related transcription factor 3 (RUNX3) (score $=0.918)$, and transforming growth factorbeta $1(\mathrm{TGF} \beta 1)($ score $=0.900)$. Several studies have focused on CDKN1A and TGF $\beta 1$, but not RUNX3, in the development of AF. Further studies should focus on the three abovementioned potentially related genes and their common polymorphisms in AF. On the contrary, the scores of related genes for PRRX1 are generally low; however, this should be verified and indicated in future research.

Although positive results were found, limitations of the current study should also be discussed. First, the literature published is relatively new, so the number of included studies is not sufficiently large based on current publications and more well-designed and larger studies in future research should be paid attention to. Second, it is possible that specific environmental and lifestyle factors influence the associations between ZFHX3 rs2106261 or PRRX1 rs3903239 polymorphism and AF including family history, age, sex, disease stage, and lifestyle. Moreover, whether the AF patients have other complications, such as hypertension, diabetes, and coronary heart disease, all the included papers have not been reported. Further comprehensive studies should include the 
abovementioned information. Third, there are several types of AF, such as persistent, permanent, pathologic, idiopathic, and paroxysmal. If enough data exist for different types of $\mathrm{AF}$ in the future, we could classify the analysis into subgroups prior to analyzing the association of the ZFHX3 rs2106261 or PRRX1 rs3903239 polymorphism with AF, which could offer more precise findings for faster translation to the clinic. Fourth, the heterogeneity was existed in our analysis, such as in total and genotype method subgroup for rs2106261 and in total for rs3903239 polymorphism. The heterogeneity for $P$ value was evaluation criteria to select the model for analysis, which may result in the final results. No publication bias was found, which may reduce the influence from the heterogeneity in our analysis.

\section{Conclusions}

Our analysis illustrated that the ZFHX3 rs2106261 and PRRX1 rs3903239 polymorphisms are associated with conspicuous AF risk in Asians. Therefore, well-designed and larger studies, including information about gene-gene/geneenvironment interactions, are recommended to confirm the abovementioned conclusions.

\section{Data Availability}

The data used to support the findings of this study are available from the corresponding author upon request.

\section{Disclosure}

The authors state this work has been presented as a preprint in Research Square according to the following link: https:// www.researchsquare.com/article/rs-117193/v1 and https:// www.researchsquare.com/article/rs-370715/v1.

\section{Conflicts of Interest}

The authors declare no conflicts of interest.

\section{Authors' Contributions}

L.W. conceived the study. M.C. searched the databases and extracted the data. W.Z. analyzed the data. L.W. wrote the draft of the manuscript. W.Z. reviewed the manuscript.

\section{Acknowledgments}

This article was supported by the Yangpu District Health and Family Planning Commission (YP18Q10) and Shanghai Yangpu District Key Discipline Project (YP19ZB03).

\section{References}

[1] X. Luo, B. Li, D. Zhang, J. Zhu, L. Qi, and Y. Tang, "Efficacy and safety of the convergent atrial fibrillation procedure: a meta-analysis of observational studies," Interactive Cardiovascular and Thoracic Surgery, vol. 28, no. 2, pp. 169-176, 2019.
[2] F. Rahman, G. F. Kwan, and E. J. Benjamin, "Global epidemiology of atrial fibrillation," Nature Reviews Cardiology, vol. 11, no. 11, pp. 639-654, 2014.

[3] X. J. Wang, P. Ding, F. Z. Wang, and Q. Liu, "Correlations of SCN5A gene polymorphisms with onset of atrial fibrillation," European Review for Medical and Pharmacological Sciences, vol. 23, pp. 7089-7097, 2019.

[4] D. Hu and Y. Sun, "Epidemiology, risk factors for stroke, and management of atrial fibrillation in China," Journal of the American College of Cardiology, vol. 52, no. 10, pp. 865-868, 2008.

[5] J. D. Roberts and M. H. Gollob, "Impact of genetic discoveries on the classification of lone atrial fibrillation," Journal of the American College of Cardiology, vol. 55, no. 8, pp. 705-712, 2010.

[6] L. Armaganijan, R. D. Lopes, J. S. Healey, J. P. Piccini, G. M. Nair, and C. A. Morillo, "Do omega-3 fatty acids prevent atrial fibrillation after open heart surgery? A metaanalysis of randomized controlled trials," Clinics (Sao Paulo), vol. 66, pp. 1923-1928, 2011.

[7] V. Fuster, L. E. Rydén, D. S. Cannom et al., "2011 ACCF/ AHA/HRS focused updates incorporated into the ACC/AHA/ ESC 2006 guidelines for the management of patients with atrial fibrillation: a report of the American college of cardiology foundation/American heart association task force on practice guidelines developed in partnership with the European society of cardiology and in collaboration with the European heart rhythm association and the heart rhythm society," Journal of the American College of Cardiology, vol. 57, no. 11, pp. e101-e198, 2011.

[8] A. Saritas, H. Kandis, D. Baltaci, and I. Erdem, "Paroxysmal atrial fibrillation and intermittent left bundle branch block: an unusual electrocardiographic presentation of mad honey poisoning," Clinics (Sao Paulo).vol. 66, no. 9, pp. 1651-1653, 2011.

[9] X. Wang, Y. Li, and Q. Li, “A comprehensive meta-analysis on relationship between CYP11B2 rs1799998 polymorphism and atrial fibrillation," Journal of Electrocardiology, vol. 52, pp. 101-105, 2019.

[10] J. B. Nielsen, R. B. Thorolfsdottir, L. G. Fritsche et al., "Biobank-driven genomic discovery yields new insight into atrial fibrillation biology," Nature Genetics, vol. 50, no. 9, pp. 1234-1239, 2018.

[11] C. Roselli, M. D. Chaffin, L. C. Weng et al., "Multi-ethnic genome-wide association study for atrial fibrillation," Nature Genetics, vol. 50, pp. 1225-1233, 2018.

[12] B. Wang, K. L. Lunetta, J. Dupuis et al., "Integrative omics approach to identifying genes associated with atrial fibrillation," Circulation Research, vol. 126, no. 3, pp. 350-360, 2020.

[13] W. Jia, X. Qi, and Q. Li, “Association between Rs3807989 polymorphism in caveolin-1 (CAV1) gene and atrial fibrillation: a meta-analysis," Medical Science Monitor, vol. 22, pp. 3961-3966, 2016.

[14] Y.-F. Jiang, M. Chen, N.-N. Zhang et al., "Association between KCNE1 G38S gene polymorphism and risk of atrial fibrillation: a PRISMA-compliant meta-analysis," Medicine, vol. 96, no. 25, Article ID e7253, 2017.

[15] Y.-Q. Zhang, Y.-F. Jiang, L. Hong, H.-J. Yang, J.-Y. Zhang, and Y.-F. Zhou, "Role of endothelial nitric oxide synthase polymorphisms in atrial fibrillation: a PRISMA-compliant meta-analysis," Medical Science Monitor, vol. 25, pp. 26872694, 2019.

[16] E. J. Benjamin, K. M. Rice, D. E. Arking et al., "Variants in ZFHX3 are associated with atrial fibrillation in individuals of 
European ancestry," Nature Genetics, vol. 41, no. 8, pp. 879-881, 2009.

[17] D. F. Gudbjartsson, H. Holm, S. Gretarsdottir et al., "A sequence variant in ZFHX3 on 16q22 associates with atrial fibrillation and ischemic stroke," Nature Genetics, vol. 41, no. 8, pp. 876-878, 2009.

[18] K. Kalinderi, N. Fragakis, M. Sotiriadou et al., "PRRX1 Rs3903239 polymorphism and atrial fibrillation in a Greek population," Hellenic Journal of Cardiology, vol. 59, no. 5, pp. 298-299, 2018.

[19] K. Ihida-Stansbury, D. M. McKean, S. A. Gebb et al., "Pairedrelated homeobox gene Prxl is required for pulmonary vascular development," Circulation Research, vol. 94, no. 11, pp. 1507-1514, 2004.

[20] E. Dolmatova, R. R. Cooper, and P. T. Ellinor, "Prrx1 interacts with and inhibits Pitx2 function," Circulation, vol. 126, p. A19452, 2012.

[21] P. T. Ellinor, K. L. Lunetta, C. M. Albert et al., "Meta-analysis identifies six new susceptibility loci for atrial fibrillation," Nature Genetics, vol. 44, no. 6, pp. 670-675, 2012.

[22] Y. Huang, C. Wang, Y. Yao et al., "Molecular basis of genegene interaction: cyclic cross-regulation of gene expression and post-GWAS gene-gene interaction involved in atrial fibrillation," PLoS Genetics, vol. 11, no. 8, Article ID e1005393, 2015.

[23] Y. Liu, B. Ni, Y. Lin et al., "The rs3807989 G/A polymorphism in CAV1 is associated with the risk of atrial fibrillation in Chinese Han populations," Pacing and Clinical Electrophysiology, vol. 38, no. 2, pp. 164-170, 2015.

[24] Y. Liu, B. Ni, Y. Lin et al., "Genetic polymorphisms in ZFHX3 are associated with atrial fibrillation in a Chinese Han population," PLoS One, vol. 9, no. 7, Article ID e101318, 2014.

[25] Y. Okubo, Y. Nakano, H. Ochi et al., "Predicting atrial fibrillation using a combination of genetic risk score and clinical risk factors," Heart Rhythm, vol. 17, no. 5, pp. 699-705, 2020.

[26] S. Tomomori, Y. Nakano, H. Ochi et al., "Maintenance of low inflammation level by the ZFHX3 SNP rs2106261 minor allele contributes to reduced atrial fibrillation recurrence after pulmonary vein isolation," PLoS One, vol. 13, no. 9, Article ID e0203281, 2018.

[27] K. T. T. Zaw, N. Sato, S. Ikeda et al., “Association of ZFHX3 gene variation with atrial fibrillation, cerebral infarction, and lung thromboembolism: an autopsy study," Journal of Cardiology, vol. 70, no. 2, pp. 180-184, 2017.

[28] G. Wells, B. Shea, D. O'Connell et al., The Newcastle-Ottawa Scale (NOS) for Assessing the Quality of Nonrandomised Studies in Meta-Analyses, Ottawa Health Research Institute, Ottawa, Canada, 2011

[29] R. DerSimonian and N. Laird, "Meta-analysis in clinical trials," Controlled Clinical Trials, vol. 7, no. 3, pp. 177-188, 1986.

[30] N. Mantel and W. Haenszel, "Statistical aspects of the analysis of data from retrospective studies of disease," Journal of the National Cancer Institute, vol. 22, pp. 719-748, 1959.

[31] Y. Hayashino, Y. Noguchi, and T. Fukui, "Systematic evaluation and comparison of statistical tests for publication bias," Journal of Epidemiology, vol. 15, no. 6, pp. 235-243, 2005.

[32] V. Napolioni, "The relevance of checking population allele frequencies and Hardy-Weinberg Equilibrium in genetic association studies: the case of SLC6A4 5-HTTLPR polymorphism in a Chinese Han Irritable Bowel Syndrome association study," Immunology Letters, vol. 162, no. 1, pp. 276-278, 2014.
[33] H.-B. Shao, K. Ren, S.-L. Gao et al., "Human methionine synthase A2756G polymorphism increases susceptibility to prostate cancer," Aging, vol. 10, no. 7, pp. 1776-1788, 2018.

[34] J. L. Anderson, J. L. Halperin, N. M. Albert et al., "Management of patients with atrial fibrillation (compilation of $2006 \mathrm{ACCF} / \mathrm{AHA} / \mathrm{ESC}$ and $2011 \mathrm{ACCF} / \mathrm{AHA} / \mathrm{HRS}$ recommendations): a report of the American College of cardiology/ American heart association task force on practice guidelines," Journal of the American College of Cardiology, vol. 61, no. 18, pp. 1935-1944, 2013.

[35] J. P. Piccini, B. G. Hammill, M. F. Sinner et al., "Incidence and prevalence of atrial fibrillation and associated mortality among Medicare beneficiaries, 1993-2007," Circulation: Cardiovascular Quality and Outcomes, vol. 5, no. 1, pp. 85-93, 2012.

[36] A. S. Go, E. M. Hylek, K. A. Phillips et al., "Prevalence of diagnosed atrial fibrillation in adults: national implications for rhythm management and stroke prevention: the anticoagulation and risk factors in atrial fibrillation (ATRIA) Study," Jama, vol. 285, no. 18, pp. 2370-2375, 2001.

[37] E. J. Benjamin, D. Levy, S. M. Vaziri, R. B. D’Agostino, A. J. Belanger, and P. A. Wolf, "Independent risk factors for atrial fibrillation in a population-based cohort. The Framingham Heart Study," Jama, vol. 271, no. 11, pp. 840-844, 1994.

[38] A. S. Gami, G. Pressman, S. M. Caples et al., "Association of atrial fibrillation and obstructive sleep apnea," Circulation, vol. 110, no. 4, pp. 364-367, 2004.

[39] E. E. Creemers, A. A. Wilde, and Y. M. Pinto, "Heart failure: advances through genomics," Nature Reviews Genetics, vol. 12, no. 5, pp. 357-362, 2011.

[40] X. Fu, X. Ma, L. Zhong, and Z. Song, "Relationship between CYP11B2-344T > C polymorphsim and atrial fibrillation: a meta-analysis," Journal of the Renin-Angiotensin-Aldosterone System, vol. 16, no. 1, pp. 185-188, 2015.

[41] J. He, W. Zhu, Y. Yu, J. Hu, and K. Hong, "Variant rs2200733 and rs10033464 on chromosome 4q25 are associated with increased risk of atrial fibrillation after catheter ablation: evidence from a meta-analysis," Cardiology Journal, vol. 25, no. 5, pp. 628-638, 2018.

[42] Y. Y. Li, L. S. Wang, and X. Z. Lu, "Mink S38G gene polymorphism and atrial fibrillation in the Chinese population: a meta-analysis of 1871 participants," TheScientificWorldJOURNAL, vol. 2014, Article ID 768681, 2014.

[43] P. Rattanawong, J. Chenbhanich, W. Vutthikraivit, and P. Chongsathidkiet, "A chromosome $4 \mathrm{q} 25$ variant is associated with atrial fibrillation recurrence after catheter ablation: a systematic review and meta-analysis," Journal of Atrial Fibrillation, vol. 10, no. 6, Article ID 1666, 2018.

[44] C. Zhai, H. Cong, Y. Liu et al., "Rs7193343 polymorphism in zinc finger homeobox 3 (ZFHX3) gene and atrial fibrillation: an updated meta-analysis of 10 case-control comparisons," BMC Cardiovascular Disorders, vol. 15, no. 1, p. 58, 2015.

[45] T. Jiang, Y.-N. Wang, Q. Qu, T.-T. Qi, Y.-D. Chen, and J. Qu, "Association between gene variants and the recurrence of atrial fibrillation: an updated meta-analysis," Medicine, vol. 98, no. 23, Article ID e15953, 2019. 